University of Nebraska - Lincoln

DigitalCommons@University of Nebraska - Lincoln

$5-16-2000$

\title{
Femtosecond luminescence dynamics in a nonlinear optical organic dendrimer
}

O. Varnavski

Wayne State University

A. Leanov

University of Nebraska - Lincoln

L. Liu

University of Nebraska - Lincoln, lliu1@unl.edu

James M. Takacs

University of Nebraska-Lincoln, jtakacs1@unl.edu

T. Goodson III

Wayne State University

Follow this and additional works at: https://digitalcommons.unl.edu/chemistrytakacs

Part of the Chemistry Commons

Varnavski, O.; Leanov, A.; Liu, L.; Takacs, James M.; and Goodson, T. III, "Femtosecond luminescence dynamics in a nonlinear optical organic dendrimer" (2000). James Takacs Publications. 2.

https://digitalcommons.unl.edu/chemistrytakacs/2

This Article is brought to you for free and open access by the Published Research - Department of Chemistry at DigitalCommons@University of Nebraska - Lincoln. It has been accepted for inclusion in James Takacs Publications by an authorized administrator of DigitalCommons@University of Nebraska - Lincoln. 


\title{
ARTICLES
}

\section{Femtosecond luminescence dynamics in a nonlinear optical organic dendrimer}

\author{
O. Varnavski \\ Department of Chemistry, Wayne State University, Detroit, Michigan 48202 \\ A. Leanov, L. Liu, and J. Takacs \\ Department of Chemistry, University of Nebraska-Lincoln, Lincoln, Nebraska \\ T. Goodson III* \\ Department of Chemistry, Wayne State University, Detroit, Michigan 48202
}

(Received 11 February 2000)

\begin{abstract}
The ultrafast intrinsic dynamics of an organic dendrimer in solution and in a thin film is reported using fluorescence upconversion spectroscopy. Femtosecond decay is detected at higher emission energies, while at lower energies a fluorescence rise time $(\sim 3 \mathrm{ps})$ was observed that is dependent on the solvent's polarity. A strong excitation energy dependence of the decay pattern was also observed. Different synthetic functional groups that comprise the macromolecular dendrimer structure were investigated. The mechanism, which describes the complex dynamics in the dendrimer system, was found to be associated with the excitation of the attached chromophore nitroaminostilbene. These results indicate the absence of excited-state interactions of functional groups within the dendrimer macromolecule. A model, which includes the existence of an intermediate nonradiative state, is proposed to describe the complex ultrafast fluorescence dynamics in the dendrimer system.
\end{abstract}

\section{INTRODUCTION}

Macromolecular structures such as conjugated organic dendrimers and polymers attract a large degree of interest in the physical sciences for a variety of reasons. Contrary to linear conjugated polymers, which are composed of linear blocks, dendrimers are synthesized by repeating branched units in a hierarchical self-similar fashion. ${ }^{1-4}$ Dendrimer macromolecules are generally characterized by their number of generations. Also, the dendrimer structure has the potential of developing branched (fractal) architectures, which could enhance the understanding of the relationship of dimensionality (geometric restriction) to excitation localization length. ${ }^{1}$ Dendrimers have received increasing attention due to their interesting electronic properties and their potential applications. ${ }^{1-7}$ The nature of optical excitations in dendrimers is still a matter of debate. It has been suggested that after excitation the electron (and hole) is localized on linear segments of the dendrimer. ${ }^{1}$ Optical excitations can be modeled as a set of weakly (Coulombic) interacting chromophores described by a Frenkel exciton Hamiltonian. ${ }^{1}$ However, the character of these interactions and the extent of delocalization of the wave function of excitons in dendrimers are not clear. Also, the character of energy migration between segments (hopping or coherent) is not certain. While no direct time-resolved ultrafast measurements were performed it was reported that direct energy transfer (and also enhancement in the light-emitting diode device efficiency) would increase with increasing generation. ${ }^{2,5}$ To clarify the character of excited-state interactions between different segments of den- drimer, time-resolved ultrafast measurements are of great necessity. However, there have been only a few reports concerning the investigations of photodynamics of dendrimer molecules, specifically photoexcitation dynamics on ultrafast (femtosecond) time scales., 8

In the present paper femtosecond time-resolved fluorescence experiments, which probe the relaxation processes in a nonlinear optical (NLO) dendrimer are reported. The present work is devoted to the very fast fluorescence dynamics of organic dendrimers for NLO and light-emitting diode applications. The dendrimer system used in this study (CZD4NSC2) contains a donor-acceptor conjugated chromophore, nitroaminostilbene (NS1), which possesses remarkable NLO properties. ${ }^{10}$ The molecular structure of the CZD4SNC2 dendrimer is shown in Fig. 1. A synthetic model compound fragment NS1 containing the nitroaminostilbene functional group and a similar dendrimer molecule CZD6NSC3 used in this study are also shown in Fig. 1. As can be seen, the macromolecule CZD4NSC2 is composed of NLO chromophores (nitroaminostilbene) and holetransporting agents (methylcarbazole). These two characteristic functional groups are very common and important for the production of NLO and photorefractive effects. ${ }^{7}$

CZD4NSC2 is a first generation dendrimer $(G 1)$ formed from diglycidyl bisphenol $A$, a molecule containing two epoxides on the terminus. This core dendrimer molecule has the functionality for further growth, and, at the same time, has the appropriate capability for the synthesis of nonlinear optical chromophore functionalization. In the second generation the bis A core is extended with a diglycidly 2,4- 

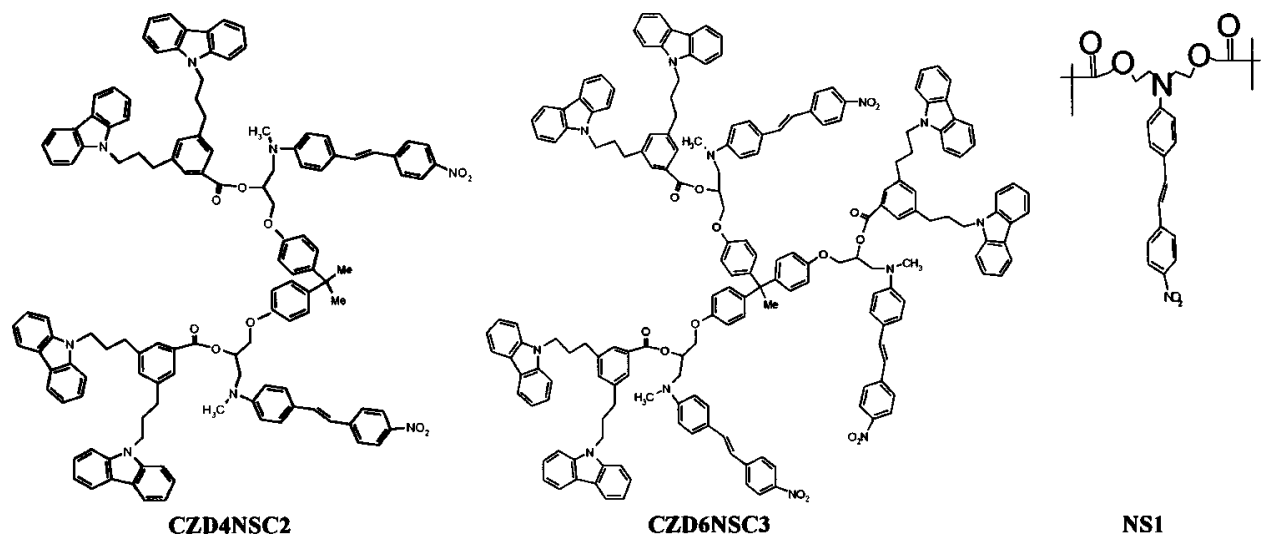

FIG. 1. The structure of the dendrimer systems CZD4NSC2, CZD6NSC3, and nitroaminostilbene (NS1) derivative used in the study.

dihroxybenzyl "branch" to afford a second generation dendrimer having four epoxides on the periphery. The initial chore dendrimer molecule has afforded the second generation dendrimer molecule, in keeping with the general definition of dendrimer formation. In the third generation there are eight epoxides, and the scheme follows for higher-order generations. While this is not the "classic" divergent dendrimer synthesis, the capacity for second, third, and fourth generation molecules have been demonstrated. CZD6NSC3 is also a first generation dendrimer formed from the corresponding tris $A$ phenol core which has also been prepared. This synthetic rational ultimately leads to hyperbranched structures, and give further justification that the initial $G 1$ molecule is seen as the core for further generation development. In order for us to properly characterize the effect of larger macromolecular architectures (increased branching or generation) it was necessary to fully investigate the smaller generation first. The dendrimer CZD4NSC2 was characterized by UVvis, infrared, and NMR spectroscopic methods (see details in Ref. 6). Our previous study of the nonlinear optical properties of these dendrimer molecules (CZD4NSC2) showed a relatively high value for the nonlinear refractive index coefficient $\left(n_{I}=1.1 \times 10^{-4} \mathrm{~cm}^{2} \mathrm{GW}\right)$ with a good figure of merit of $0.02{ }^{6}$

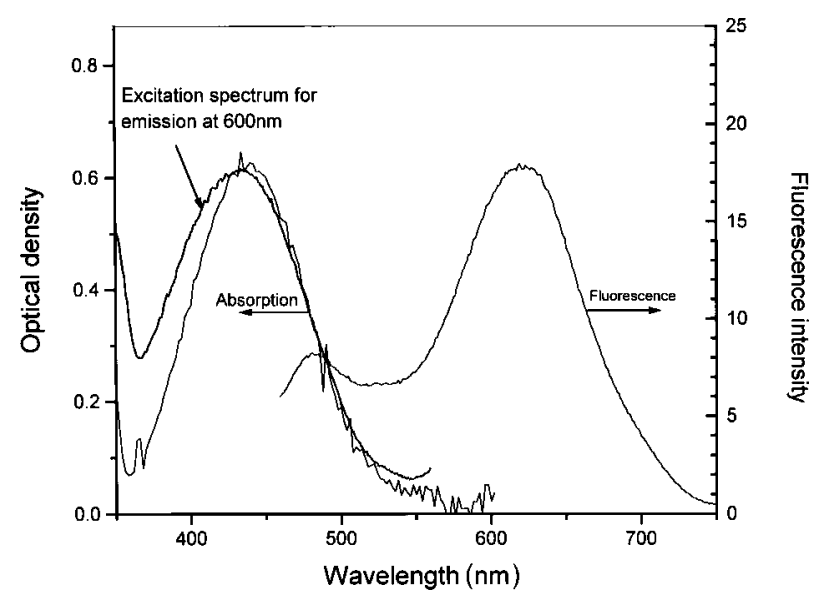

FIG. 2. Steady-state absorption and emissin spectra $\left(\lambda_{\text {exc }}=450\right.$ $\mathrm{nm})$, and 0 spectrum for emission at $600 \mathrm{~nm}$ of the dendrimer CZD4NS2 in chloroform.

\section{EXPERIMENT}

The steady-state absorption was measured with an Ocean Optics fiber optical spectrometer and a Hewlett-Packard $8452 \mathrm{~A}$ spectrometer. The steady-state emission measurements were taken on a Shimadzu R1501 spectrofluorophotometer. Continuous-wave photoluminescence, excitation spectrum of emission at $600 \mathrm{~nm}$, and absorption spectra of the dendrimer CZD4NSC2 solution in chloroform are shown in Fig. 2. The absorption spectra show a peak at $437 \mathrm{~nm}$ for all solvents used in this study. On the other hand, we found the emission spectra (the shape and max position) to be solvent dependent. For example, the emission maximum is measured in chloroform to be at $625 \mathrm{~nm}$ with a shoulder peak at about $485 \mathrm{~nm}$, while for chlorobenzene solution the fluorescence spectrum peaks at $600 \mathrm{~nm}$ and has no shoulder. The fluorescence maximum in different solvents showed a solvatochromic shift in accordance with the solvent polarity while the appearance of shoulder could be indicative of dual fluorescence. ${ }^{11-13}$

For fast fluorescence kinetic measurements we apply the femtosecond fluorescence upconversion technique for time resolved detection of the luminescence. ${ }^{14}$ The optical arrangement for the upconversion experiments was described previously. ${ }^{6,15}$ The laser source was a Ti:sapphire laser with an average pulse width of $100 \mathrm{fs}$ tuned at $790-860 \mathrm{~nm}$, and a repetition rate of $82 \mathrm{MHz}$ (Tsunami, Spectra Physics). The sample was excited with light pulses delivered by a frequency-doubled output of the laser at $395-430 \mathrm{~nm}$. The fluorescence emitted from the sample was focused with an achromatic lens into a non-linear crystal made of betabarium borate. A temporal profile of the fluorescence was monitored by sum-frequency generation with reference pulse from the laser at 790-860 nm that was first passed through a variable delay line. Sum-frequency light was dispersed by a monochromator and detected by a single-photon-counting system. The time resolution was determined by the pulse width of the laser and group velocity dispersion within optical elements of the system. The full width at half maximum of the cross-correlation function at 790/395 nm was estimated to be 190 fs. It is important to note that we could observe the fluorescence dynamics on a time scale covering almost four decades ( $200 \mathrm{fs}-1 \mathrm{~ns}$ ) in one scan. All experiments presented in this paper were performed at room temperature. The rotating sample cell and holder (1-mm thick- 


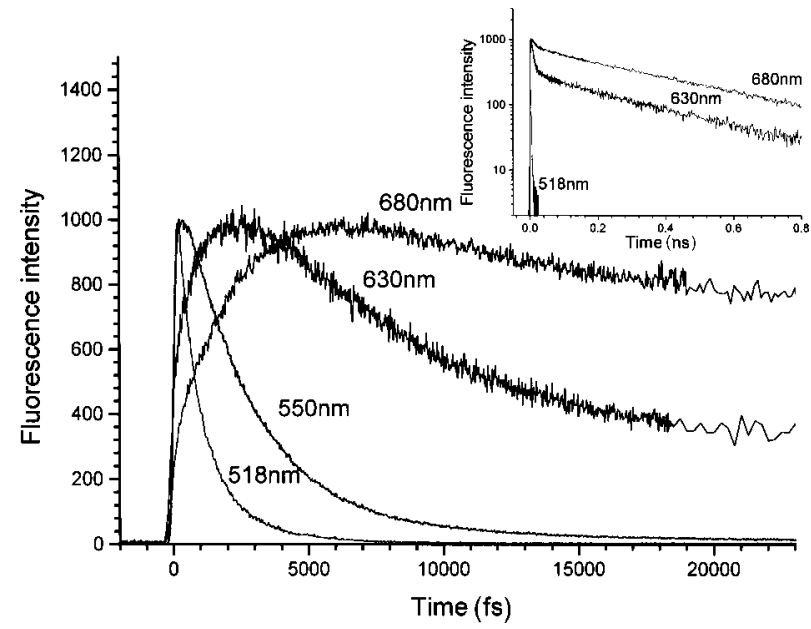

FIG. 3. Fluorescence dynamics of the dendrimer CZD4NS2 in chloroform for different emission wavelengths, when excited at 395 nm. Inset-long-time-scale decays.

ness in the case of solutions, and $\sim 1-\mu \mathrm{m}$ thickness in the case of a thin film) were used to avoid thermal and photochemical accumulative effects. Pure spectroscopic grade solvents where used and checked for impurities by nuclear magnetic resonance spectroscopy. The average concentration used in our investigations was $\sim 2 \times 10^{-4} \mathrm{M}$. It should be noted that there was no detectable concentration of the fluorescence dynamics reported here in the range from $2 \times 10^{-4}$ to $2 \times 10^{-3} \mathrm{M}$. The excitation average power was kept at the level of a few (in most cases $\sim 1 \mathrm{~mW}$ ) milliwatts. This corresponds to input pulse energy well below $0.1 \mathrm{~nJ}$ at $395 \mathrm{~nm}$. We found that there was no excitation intensity dependence of the decay dynamics when input powers were below $10 \mathrm{~mW}$.

\section{RESULTS AND DISCUSSION}

Shown in Fig. 3 is the result of ultrafast upconversion measurements of the femtosecond fluorescence relaxation processes in CZD4NSC2 at different emission wavelengths. The excitation wavelength in this case was $395 \mathrm{~nm}$. It is clearly seen that the fluorescence dynamics of this system (on a time scale ranging over four decades) is rather complicated, and cannot be described by a simple exponential decay law. We also noticed a very strong dependence of the decay pattern on emission wavelength. As can be seen from Fig. 3, there is a very fast decay in the case of the $518 \mathrm{~nm}$ result detected. At this emission wavelength the experimental traces could be satisfactory fitted by a biexponential equation with two decay times $\tau_{1}$ and $\tau_{2}$ and pre-exponential factors $A_{1}$ and $A_{2}$. These parameters were found to be $\tau_{1}$ $=1297 \mathrm{fs}, A_{1}=1342, \tau_{2}=3253 \mathrm{fs}$, and $A_{2}=1342$, and $\tau_{1}$ $=234 \mathrm{fs}, A_{1}=694, \tau_{2}=527 \mathrm{fs}$, and $A_{2}=248$ for chloroform and dichloromethane solutions, respectively. However, for longer emission wavelengths there is a real fluorescence rise time followed by a very long (nanosecond) decay. Note that the decay time of the long-lived component does not depend on the emission wavelength. However, the relative contribution (amplitude) of this component does depend on the emission wavelength (see the inset of Fig. 3). The fluorescence decay pattern (rise time plus emission-wavelength-dependent

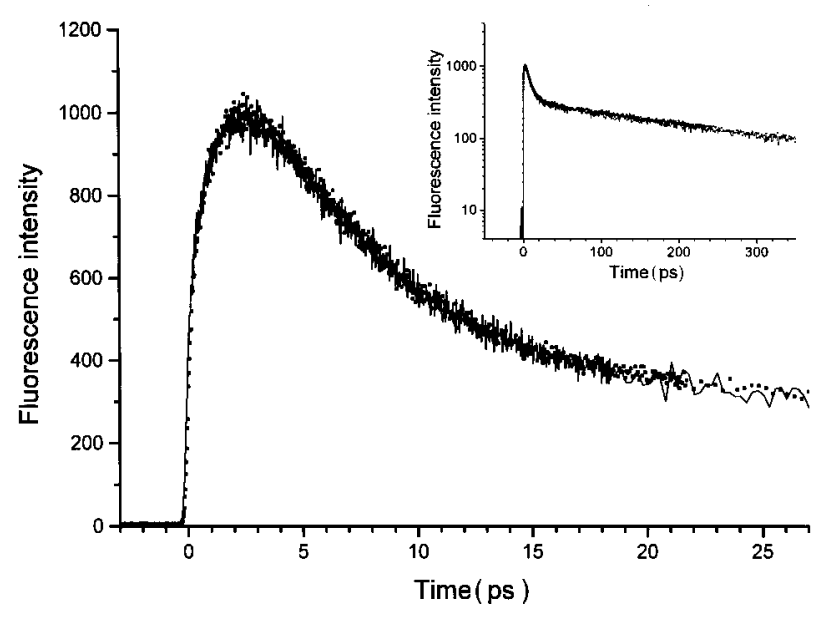

FIG. 4. Dynamics of the emission of the dendrimer CZD4NS2 (scatter graph) and nitroaminostilbene derivative (NS1) (solid line) at $630 \mathrm{~nm}$, in chloroform. Inset-long-time-scale decays. The excitation wavelength is $395 \mathrm{~nm}$.

contribution of the long-lived component) seen for the CZD4SNC2 dendrimer is typical of two-component (dual) fluorescence. $^{11-13,16,17}$ The presence of a blue shoulder at about $485 \mathrm{~nm}$ in the steady-state fluorescence spectrum shown in Fig. 2 also supports this dual fluorescence model. Generally, dual (multiple) fluorescence could be associated with either solvent rearrangement ${ }^{16,18}$ or photoinduced solute conformational changes. ${ }^{11,13,17}$ It might also be suggested that the long-lived decay and the rise-time components could be connected with the formation of aggregates such as excimers or exciplexes. Note that the existence of excimerlike interactions was invoked to explain the fast excited-state dynamics of another type of dendrimer macromolecule bases on phenylene building blocks. ${ }^{8,9}$

To test the possibility of intramolecular interactions such as energy transfer or excimerlike formation, we investigated several isolated cases with dendrimer structures containing synthetic fragments (components) of either carbazole or nitroaminostilbene (NAS) functional groups. In the last case we measured the fluorescence dynamics and steady-state spectra for model compounds containing either one (NS1, Fig. 1) or two attached NAS chromophore groups (for the case of two NAS groups on a dendrimer fragment, it could be suggested that there is a possibility of bicimer formation ${ }^{19}$ due to the short distance between the two NAS chromophore groups). We also measured the fluorescence kinetics for a more complicated dendrimer molecule CZD6NSC3 containing three NAS groups and three carbazole groups. In this molecule one may expect enhanced intersegment interactions due to the reduced distances between chromophore groups.

However, fluorescence dynamic measurements performed with all the systems mentioned above showed that there is no influence of the other functional group (carbazole) or the second NAS group on the dynamics of the dendrimer system. In particular, we found no detectable difference in the rather complex fluorescence kinetics of CZD6NSC3 and CZD4NSC2 dendrimer systems. Comparing the fluorescence kinetics of a CZD4NSC2 dendrimer excited at 390-430 nm with that of the chromophore NS1, we found this kinetics to be strongly directed by the dynamics of NS1. The similarity in the fluorescence dynamics of the dendrimer macromol- 


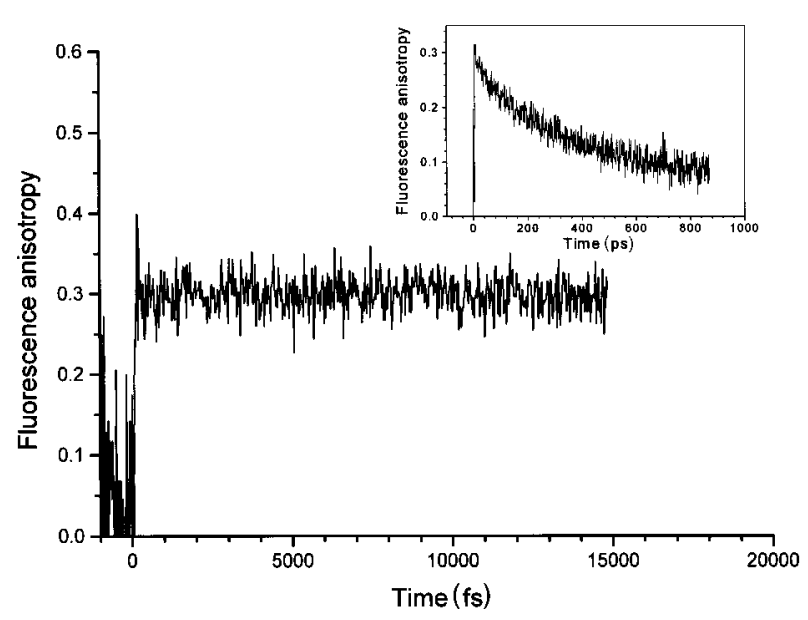

FIG. 5. Time-resolved fluorescence anisotropy of the CZD4NS2 dendrimer at $630 \mathrm{~nm}$. Inset - the long-time-scale decay of fluorescence anisotropy. The excitation wavelength is $395 \mathrm{~nm}$.

ecule CZD4NSC2 with that of the NS1 chromophore is demonstrated in Fig. 4.

We investigated the concentration dependence, intensity dependence, and solvent polarity dependence of the small synthetic fragment of the organic dendrimer, and in all cases the fluorescence kinetics on a time scale covering four decades showed no detectable difference from that obtained for the entire dendrimer molecule. It is also interesting to note that the fast nonlinear response was found to be the same for NS1 and CZD4NSC2. ${ }^{6}$ From all these results it can be concluded that the complex fluorescence dynamics shown in Figs. 3 and 4 (the long-lived decay and the rise-time components) is not connected with the formation of excited-state aggregates (excimers or exciplexes), as was observed for another type of dendrimer molecule composed of phenylene building blocks. ${ }^{8,9}$ To check the possibility of excitation energy migration between differently oriented chromophores within dendrimer macromolecules we measured the timedependent fluorescence anisotropy. Generally, a fast fluorescence depolarization could be an indication of the energy migration between differently oriented chromophores. ${ }^{20-23}$ Shown in Fig. 5 is the result of ultrafast upconversion measurements of the fluorescence anisotropy in a CZD4SNC2 dendrimer at $630 \mathrm{~nm}$. No fast fluorescence depolarization in the time range $200 \mathrm{fs}-50 \mathrm{ps}$ was found in our case. The relatively slow depolarization shown in the inset of Fig. 5 is connected with the overall rotational diffusion of the dendrimer molecule in chlorobenzene $\left(\tau_{R} \approx 600 \mathrm{ps}\right)$. These fluorescence depolarization results give additional support to the lack of pronounced interchromophore excited-state energy transfer (interaction) in our dendrimer macromolecule. Thus our steady-state and time-resolved measurements showed that the electronic excitation in CZD4NSC2 and CZD6NSC3 dendrimer systems is localized on one NAS chromophore, and that the complex excited-state dynamics is entirely directed by intrachromophore processes.

Although there have been a great many publications concerned with the optical properties of donor-acceptor stilbene derivative, the discussion concerning the intramolecular charge transfer and the involvement of "twisted" or conformational relaxed states is still very active. ${ }^{11-13,16,24}$ The lowlying electronic states of donor-acceptor substituted stilbenes

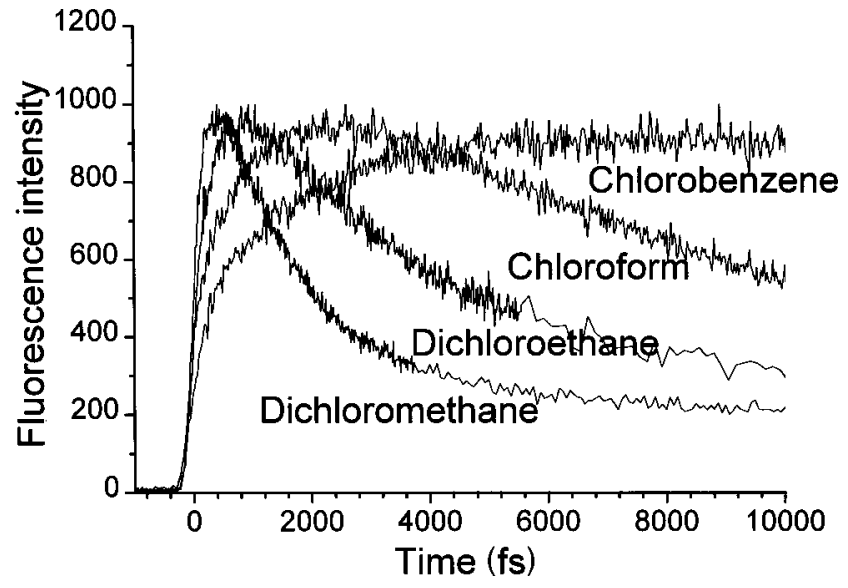

FIG. 6. Dynamics of the emission of the dendrimer in different solvents at $630 \mathrm{~nm}$. The excitation wavelength is $395 \mathrm{~nm}$.

have a high degree of charge-transfer character. The degree of intramolecular charge transfer can be enhanced by polar solvent stabilization accompanied by nuclear reorganization. An interpretation that relates the photophysics of nitroaminostilbenes to the formation of twisted intramolecular charge transfer (TICT) states was suggested. The TICT states are associated with a twisting of various single and double bonds in the molecule. ${ }^{11}$ Therefore, the stabilization dynamics of the intramolecular charge-transfer state should reflect the balance between intramolecular energy relaxation and solvent reorganization. The dynamics is expected to be rather complex. However, the experimental proof of one or other theoretical models for NAS photophysics relied mainly on the concepts of fluorescence quantum yield and mean excited-state decay time. ${ }^{11,12}$ At the same time, measurements with a femtosecond time resolution performed for related donor-acceptor dimethylaminocyanostilbene showed complicated dynamics with a rise-time component and several decay time components, which obviously could not be described in terms of a simple decay time concept. However, no time-resolved fluorescence measurement with femtosecond time resolution has been published for nitroaminostilbene derivatives. Shown below are results of ultrafast fluorescence measurements for NS1, a part of the dendrimer CZD4SNC2. We have already demonstratred that the NS1 strongly directs the fluorescence dynamics of the dendrimer CZD4SNC2. It was shown above (Fig. 3) that for our system containing NAS we detected a very strong dependence of the decay pattern with emission wavelength. In general, such dynamics are typical of multiple fluorescence state emission when the formation of relaxed species with a simple precursor-successor relationship takes place. ${ }^{19}$ However, the solvent and excitation wavelength effects on the dynamics provided a more complex dynamical diagram for our dendrimer system.

We performed ultrafast fluorescence measurements in thin films and in different solvents of polarity and viscosity. The relaxation profile was found to correlate extremely well with the solvent's polarity, but not on the solvent's viscosity. Shown in Fig. 6 is the solvent polarity dependence of the fluorescence rise time, which probed at a wavelength of 630 $\mathrm{nm}$. As can be seen in Fig. 6, there were four different solvents tested. These solvents varied in polarity in the 
order dichloromethane $>$ dichloroethane $>$ chloroform $>$ chlorobenzene. ${ }^{25}$ Similar measurements with nonhalogenated solvents were also performed, and the NMR spectra for the dendrimer solutions showed no chemical destruction or photochemical instability. It is important to note that there were no accumulative effects due to repeated laser excitation of the dendrimer macromolecule. At shorter time scales (less than 20 ps), it can clearly be seen that the rise time of the luminescence increases with decreasing polarity. On the other hand, for the long-lived component of the decay the lifetime was found to be weakly dependent on the solvent polarity. However, the contribution (or amplitude) of the long-lived component increases strongly with decreasing solvent polarity.

We also measured a thin film (spin coated from a chloroform solution of the dendrimer), and the results were the same as those obtained from the most polar solvent. At shorter emission wavelengths (such as $518 \mathrm{~nm}$ ) the fluorescence kinetics showed a multiexponential character where no satisfactory fit to one-exponential decay law was obtained. The mean relaxation time of the fast decay results obtained at lower emission wavelengths (518 $\mathrm{nm}$, for example) showed the same solvent polarity trend as that obtained for the rise time detected at longer emission wavelengths. In both cases the increase in polarity caused the relaxation times to decrease and the reduction (elimination) of the risetime effect. In many cases this is indicative of a precursorsuccessor relationship between two fluorescent states. ${ }^{11,16}$ Such a dependence of the fluorescence kinetics on the solvent polarity at particular emission wavelengths could be the result of a solvatochromic shift of the fluorescence line, which is quite strongly dependent on polarity for donoracceptor systems like NAS. ${ }^{11-13,16}$ However, a comparison of the fluorescence dynamics at the fluorescence maxima also showed a dependence on polarity (the same trend as for fixed emission wavelength). This suggests that the dynamics of the relaxed state formation process are also polarity dependent, and indicates that observed solvent-dependent dynamics are not directed by the solvatochromic shift.

The results reported above for the CZD4NSC 2 dendrimer correlate well with the formation of multiple fluorescence states originating from one excited state, as described by a simple precursor-successor relationship. ${ }^{11}$ This model is adequate for the formation of excited states with conformational changes involving the twisting of one of the single bonds ("twisted intermolecular charge transfer" TICT states), ${ }^{11}$ as well as for solvent relaxation processes. ${ }^{16}$ In our results, the emission wavelength dependence of the short lifetime component and the contribution of the long-lived component are in accordance with the formation of two fluorescence states. Also, the polarity dependence of the short lifetime component as well as the long-lived component contribution do not contradict this model. However, our results concerning the excitation wavelength dependence shown in Fig. 7 do not support this simple precursor-successor scheme. In our measurements the excitation wavelength was varied from 395 to $430 \mathrm{~nm}$. The results showed the main trend of a decrease of short relaxation times with an increase of the excitation wavelength. The contribution of the longlived component at emission wavelengths of 600-680 nm decreased with an increase of the excitation wavelength,

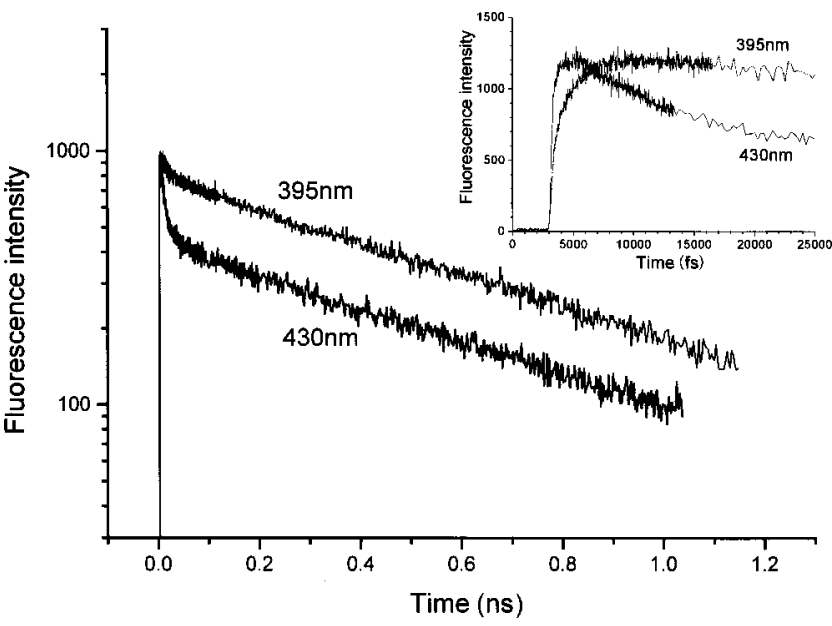

FIG. 7. Fluorescence dynamics of the dendrimer CZD4NS2 in chloroform for different excitation wavelengths, when detected at $630 \mathrm{~nm}$. Inset-short-time-scale dynamics.

while the relaxation time of the long-lived component was found to be independent of the excitation wavelength. For example, the emission of the chlorobenzene solution at 518 $\mathrm{nm}$ gave decay times that were estimated at $\sim 2.5 \mathrm{ps}$ and 880 fs (monoexponential fits) for excitations at 395 and $430 \mathrm{~nm}$, respectively. The same is true for rise times at longer emission wavelengths such as $630 \mathrm{~nm}$ : they were estimated to be $\sim 2.3 \mathrm{ps}$ and $665 \mathrm{fs}$ for excitations at 395 and $430 \mathrm{~nm}$ respectively (Fig. 7, inset). The increase of the long-lived redshifted component contribution with the increase of excitation photon energy shown in Fig. 7 correlates well with the blueshift of the excitation spectra for emission at $600 \mathrm{~nm}$. The blueshift of the excitation spectrum with respect to the absorption spectrum indicates an additional channel of the excitation of redshifted fluorescence via high-lying thermally nonequilibrated states. This trend does not correlate with a simple precursor-successor model, which implies the existence of two fluorescent states (species) originating from one relaxed excited state.

We have observed the greater contribution of long-lived redshifted emission when we excite the system with higherenergy photons. At the same time the rise-time component increases with the increase of excitation photon energy (see Fig. 7, inset). The increase of the fluorescence rise time with the decrease of excitation wavelength for another system PYRBN ( $N$-pyrolidinyl-benzonitrile) with charge transfer state was explained in the framework of barrierless stochastic staircase model of charge-transfer process. ${ }^{26,27}$ An important feature of barrierless reactions is that the relaxation of the excited state may depend strongly on the initial conditions, ${ }^{27}$ which is the case in our experiment. However, our results do not correlate with this model at least for two reasons. The first can be seen by the observed drop of the charge-transfer state contribution approaching the sink position [increasing the excitation wavelength (Fig. 7)], which is quite difficult to explain in the frame of the main assumptions of this model. ${ }^{26,27}$ Second, according to the barrierless model the reaction should be under the complete control of the solvent viscosity, which is not the case in our system. We found the relaxation profile to correlate quite well with the solvent's polarity, but not on the solvent's viscosity. 


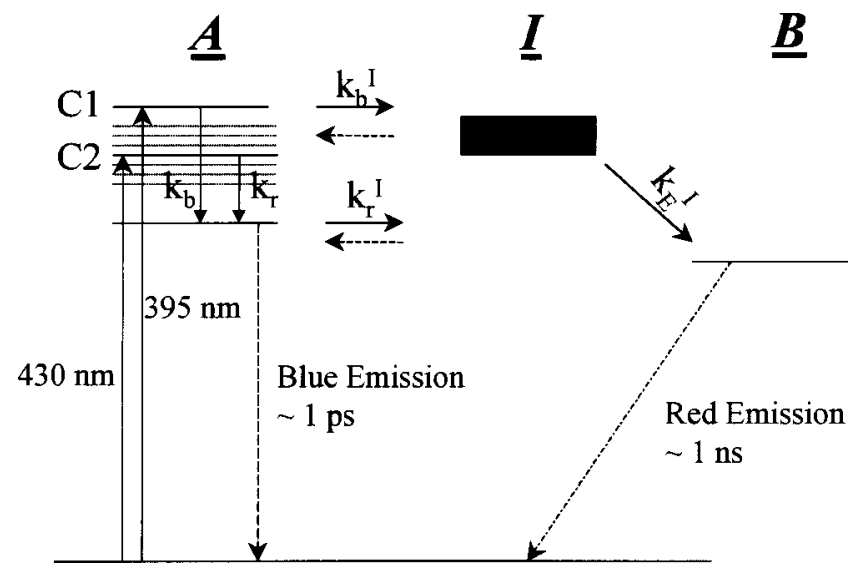

FIG. 8. Suggested energy levels and kinetic diagram (the nonradiative decay channel is not shown; see the text).

For the case of the solvent relaxation control of the charge-transfer process, there is only one state in the system that changes its charge-transfer character gradually on the time scale of the solvent relaxation. The presence of two distinct peaks in the fluorescence spectra (Fig. 2) along with the nontrivial dependence of the fluorescence dynamics on excitation wavelength (Fig. 7) makes complete solvent control of the observed process quite improbable. Intramolecular dynamics such as large-amplitude motions (conformational movements) and crossing of the thermal barrier should be taken into account in our case.

A phenomenological model illustrated in the diagram in Fig. 8 (inset) can be proposed for our system. Initially we excite the molecule with excess vibrational energy to states $\mathrm{C} 1$ or $\mathrm{C} 2$ within a manifold denoted as $A$. These states are created by excitation at 395 and $430 \mathrm{~nm}$, respectively. The manifold $A$ may correspond to the highly polar excited species of NAS with a planar conformation. ${ }^{11}$ Several relaxation channels are possible for the Frank-Condon states initially formed. The first channel is a fast vibrational relaxation accompanied by solvent rearrangement, and is given the rate constant $k_{b}$ or $k_{r}$ depending on the excitation wavelength. The second channel is connected with the formation of the long-lived (1-ns) fluorescence species, which is suggestively due to the TICT states of the stilbene chromophore. ${ }^{11}$ The formation of the second channel is strongly dependent on the decay of an intermediate (denoted $I$ ) trap state and is relatively long ( $\sim 3 \mathrm{ps}$, rate constant $\left.k_{E}^{I}\right)$ as seen in our rise-time results. The intermediate trap is necessary because the relaxation rates $\left(k_{b}\right.$ and $\left.k_{r}\right)$ must be comparable to the formation rates of $k_{b}^{I}$ and $k_{r}^{I}$ in order to produce an appreciable amount of species $A$ and $B$. The relaxation rates $k_{b}$ and $k_{r}$ must be faster than $200 \mathrm{fs}$, due to the absence of any detectable rise time for blue emission (we should note here that the thermal population of upper states is negligible). Thus the large dif- ference in rate constants $k_{b}^{I}$ (or $k_{r}^{I}$ ) and $k_{E}^{I}$ indicates the existence of such an intermediate trap state. Also, due to the excitation dependence of the contribution of species $A$ (where excitation at $395 \mathrm{~nm}$ gives a larger contribution of $A$ ) the rate constant $k_{b}^{I}$ should be much larger than $k_{r}^{I}$. That could be either due to the barrier crossing or due to the transition to the additional conformational state lying slightly above the planar conformation state $A$. The presence of several states associated with the twisting around a single bond has been shown for dimethylaminonitrostilbene by use of quantum-chemical calculations. ${ }^{11}$ These states $\left(A_{i}^{*}\right.$, as in Ref. 11) could be either radiative or nonradiative depending on the bond position, twisting angle, and particular energy level. ${ }^{11}$ It was suggested that the relaxed state is one of the radiative TICT states to which the molecule relaxes after excitation to the planar conformation. This model was supported by indirect experiments on fluorescence quantum yield ${ }^{11}$ and on bridged compounds. ${ }^{13}$ Our direct femtosecond dynamics studies showed that a nonradiative trap state $(I)$ is indeed necessary. One of the nonradiative TICT sates $\left(A^{*}\right)$ lying slightly above the planar configuration level $A$ (in our diagram) could be the intermediate state $I$. The final longlived state $B$ may be associated with a different radiative TICT state. It should be noted that possible nonradiative channels of excited-state relaxation were not discussed in the proposed diagram. These processes can be associated with such mechanisms as rotation around double bonds (trans-cis isomerization) and the nonradiative channel connected to nitro group. ${ }^{11-13}$ The nonradiative processes strongly influence the absolute values of the decay times and the fluorescence quantum yield. We assume that these nonradiative processes occur simultaneously with the relaxation processes described above.

\section{CONCLUSIONS}

In conclusion, we have measured the intrinsic ultrafast fluorescence dynamics of an organic NLO dendrimer macromolecule. No indication of excited-state interactions between different nitroaminostilbene segments of a dendrimer have been found, even for the case of different dendrimer structures with different interaction lengths. The fast fluorescence dynamics was completely directed by a nitroaminostilbene fragment of a dendrimer. The mechanism describing the CZD2NSC4 dynamics was found to be inconsistent with a simple precursor-successor scheme when multiple fluorescence states originate from one relaxed excited state. From the kinetics of the formation and decay of fluorescent species, an intermediate trap state was found to be necessary in order to completely describe our results. This intermediate state and one of the fluorescence states were assigned to nonradiative and radiative states, and are associated with a twisting around the single bond of the nitroaminostilbene group in the organic dendrimer system.

\footnotetext{
*Author to whom all correspondence should be addressed. Electronic address: tgoodson@ chem.wayne.edu

${ }^{1}$ S. Tretiak, V. Chernyak, and S. Mukamel, J. Chem. Phys. 110, 8161 (1999).

${ }^{2}$ R. Kopelman, M. Shortreed, Zhong-You Shi, W. Tang, Zhifu Xu, J. Moore, A. Bar-Haim, and J. Klafter, Phys. Rev. Lett. 78, 1239
}

(1997).

${ }^{3}$ D. A. Tomalia, A. M. Naylor, and W. A. Goddar III, Angew.

Chem. Int. Ed. Engl. 29, 138 (1990).

${ }^{4}$ J. M. J. Frechet, Science 263, 1710 (1994).

${ }^{5}$ M. Halim, J. N. G. Pillow, I. D. W. Sammuel, and P. L. Burn, Adv. Mater. 11, 371 (1999). 
${ }^{6}$ O. Varnavski, A. Leanov, L. Liu, J. Takacs, and T. Goodson III, J. Phys. Chem. B 104, 179 (2000).

${ }^{7}$ Y. D. Zhang, T. Wada, and H. Sasabe, J. Mater. Chem. 8, 809 (1998).

${ }^{8}$ J. Hofkens, L. Larettini, G. DeBelder, T. Gensch, M. Maus, T. Vosch, Y. Karni, G. Schweitzer, F. C. DeSchryver, A. Herrmann, and K. Mullen, Chem. Phys. Lett. 304, 1 (1999).

${ }^{9}$ Y. Karni, S. Jordens, G. DeBelder, G. Schweitzer, J. Hofkens T. Gensch M. Manus, F. C. DeSchryver, A. Herrmann, and K. Mullen, Chem. Phys. Lett. 310, 73 (1999).

${ }^{10}$ T. Goodson III and C. H. Wang, J. Phys. Chem. 100, 1392 (1996).

${ }^{11}$ R. Lapouyade, A. Kuhn, J-F. Letard, and W. Rettig, Chem. Phys. Lett. 208, 48 (1993).

${ }^{12}$ H. Goerner, Ber. Bunsenges. Phys. Chem. 102, 726 (1998).

${ }^{13}$ J.-F. Letard, R. Lapouyade, and W. Rettig, Chem. Phys. 186, 119 (1994).

${ }^{14}$ H. Mahr and M. D. Hirch, Opt. Commun. 13, 96 (1975).

${ }^{15}$ O. Varnavski and T. Goodson III, Chem. Phys. Lett. 320, 688 (2000).

${ }^{16}$ N. Eilers-Koenig, T. Kuhne, D. Schwarzer, P. Voehringer, and J.
Schroeder, Chem. Phys. Lett. 253, 69 (1996).

${ }^{17}$ B. Strehmel, H. Seifert, and W. Rettig, J. Phys. Chem. 101, 2232 (1997).

${ }^{18}$ M. L. Horng, J. A. Gardecki, A. Papazyan, and M. Maroncelli, J. Phys. Chem. 99, 17311 (1995).

${ }^{19}$ E. Gilabert, R. Lapoyade, and C. Rulliere, Chem. Phys. Lett. 185, 82 (1991).

${ }^{20}$ L. Larettini, G. DeBelder, G. Schweitzer, M. Van der Auweraer, and F. C. DeSchryver, Chem. Phys. Lett. 295, 11 (1998).

${ }^{21}$ R. Jimenez, S. N. Dikshit, S. E. Dradforth, and G. R. Fleming, J. Phys. Chem. B 100, 6825 (1996).

${ }^{22}$ Y. R. Kim, M. Lee, J. R. G. Thorne, and R. M. Hochstrasser, Chem. Phys. Lett. 145, 75 (1988).

23 O. Varnavski, G. Menkir, P. Burn, and T. Goodson III (unpublished).

${ }^{24}$ F. D. Lewis, R. S. Kalgutkar, and J.-S. Yang, J. Am. Chem. Soc. 121, 12045 (1999).

25 C. Reichardt, Solvents and Solvent Effects in Organic Chemistry (VCH, Weinheim, 1990).

${ }^{26}$ B. Bagchi, Chem. Phys. Lett. 135, 558 (1987).

${ }^{27}$ B. Bagchi and G. R. Flemming, J. Phys. Chem. 94, 9 (1990). 